\title{
Cinética de hidratação de ligantes à base de alumina hidratável ou aluminato de cálcio
}

\section{(Kinetics of hydration of binders based on hydratable alumina or calcium aluminate cement)}

\author{
I. R. Oliveira, J. R. Garcia, V. C. Pandolfelli \\ Departamento de Engenharia de Materiais - DEMa, Universidade Federal de S. Carlos - UFSCar \\ Rod. Washington Luiz, km 235, C.P. 676, S. Carlos, SP 13565-905 \\ ivone@iris.ufscar.br,vicpando@power.ufscar.br \\ vicpando@power.ufscar.br
}

\begin{abstract}
Resumo
O estado de dispersão da matriz de um concreto refratário apresenta uma grande influência no comportamento reológico desse material, determinando as técnicas utilizadas para a sua aplicação. Tais métodos normalmente exigem a preparação de concretos com elevada fluidez, que possam ser bombeados com facilidade e sejam capazes de preencher moldes de formato complexo sem a necessidade de aplicação de vibração. Entretanto, embora tais requisitos favoreçam uma boa trabalhabilidade do concreto, tendem a aumentar o tempo requerido para efetuar a desmoldagem do corpo conformado. Uma vez que o desenvolvimento da resistência mecânica do concreto está intimamente relacionado ao processo de hidratação do ligante hidráulico, este necessita ser controlado quando se busca a redução do tempo para a desmoldagem. Tal controle depende de um profundo conhecimento das variáveis que determinam a cinética das reações. Neste contexto, o objetivo deste trabalho foi o de avaliar a influência do tipo de ligante hidráulico, da temperatura e da presença de finos (matriz) ou de aditivos inorgânicos adicionados ao concreto sobre o processo de hidratação por meio de medidas de temperatura e ensaios reológicos oscilatórios em função do tempo.
\end{abstract}

Palavras-chave: hidratação, alumina hidratável, aluminato de cálcio.

\begin{abstract}
The dispersion of refractory castables matrix presents a great influence on their rheological behavior, which defines the most appropriate methods for placing these materials. The growing demand for automatically transported refractory castables has promoted the use of pumpable castables, usually specified as self flow compositions. Nevertheless, castables with higher fluidity present longer workability, leading to extended demoulding times. Because the strength development is intimately linked to the hydration process of calcium aluminate cement or hydratable alumina, it needs to be controlled in order to reach the minimum time for demoulding, contributing to reducing overall costs. The control of cement hydration depends on the knowledge of the variables that determine the kinetics of the involved reactions. In this context, the objective of this work was to evaluate the influence of the sources of the hydraulic binder, the temperature and the presence of matrix or inorganic additives on the hydration process, carried out through temperature measurements and oscillatory rheological tests, as a function of the time.
\end{abstract}

Keywords: hydration, hydratable alumina, calcium aluminate cement.

\section{INTRODUÇÃO}

A aplicação de concretos refratários, principalmente na siderurgia, é um processo em constante evolução, que apresenta uma forte dependência dos avanços dos conhecimentos sobre os ligantes hidráulicos. Isso se deve a influência exercida pelos ligantes sobre as propriedades reológicas e desenvolvimento de resistência mecânica de concretos, que por sua vez, são responsáveis pela trabalhabilidade e tempo de desmoldagem desses materiais.

Os ligantes hidráulicos são em sua maioria, óxidos capazes de se rehidratar e promover a coesão do corpo conformado. Nesta categoria podem ser incluídos os cimentos de alta alumina e as aluminas hidratáveis.

Os cimentos de alta alumina (cimentos de aluminato de cálcio, CAC) constituem os agentes ligantes mais utilizados em aplicações industriais, devido principalmente a sua disponibilidade, baixo custo, capacidade de conferir alta resistência mecânica a verde aos concretos e resistência ao ataque de agentes agressivos, quando em uso [1].

O processo de hidratação das partículas de cimento em água envolve três períodos distintos: dissolução de íons, nucleação e precipitação de fases hidratadas. Tão $\log$ as partículas de cimento entram em contato com a água, fases anidras de aluminato de cálcio começam a dissociar e liberam íons cálcio $\left(\mathrm{Ca}^{2+}\right)$ e tetra hidróxido aluminato $\left(\mathrm{Al}(\mathrm{OH})_{4}^{-}\right)$ no meio líquido. $\mathrm{O}$ processo de dissolução ocorre até que 
a concentração desses íons na solução aquosa alcance um certo nível de saturação, promovendo a sua precipitação na forma de hidratos de aluminato de cálcio, por meio de mecanismos de nucleação e crescimento. A precipitação dos primeiros produtos hidratados diminui a concentração de íons em solução para níveis abaixo da condição de saturação, favorecendo a dissociação de fases anidras. Isso resulta num processo contínuo de dissolução-precipitação que ocorre até que a maioria (ou toda) fase anidra tenha reagido. Desde que a nucleação heterogênea na superfície das partículas seja favorecida, as fases hidratadas precipitadas tendem a formar fortes ligações entre partículas vizinhas, promovendo o endurecimento ou pega do concreto $[2,3]$.

Os cimentos aluminosos comerciais são constituídos principalmente pelas fases: $\mathrm{CaO} \cdot \mathrm{Al}_{2} \mathrm{O}_{3}(\mathrm{CA})$, cerca de $40 \mathrm{a}$ $70 \%$ do produto; $\mathrm{CaO} .2 \mathrm{Al}_{2} \mathrm{O}_{3}\left(\mathrm{CA}_{2}\right)$, que é a segunda em proporção $(<25 \%)$ e a fase $12 \mathrm{CaO} .7 \mathrm{Al}_{2} \mathrm{O}_{3}\left(\mathrm{C}_{12} \mathrm{~A}_{7}\right)$, em teores de $3 \%$ ou menos. A fase $\mathrm{CA}_{2}$ é a mais refratária das três, requerendo um longo tempo para completar sua hidratação. Já a fase $\mathrm{C}_{12} \mathrm{~A}_{7}$, ao contrário, possui baixa refratariedade, entretanto, necessita de um tempo curto para se hidratar e pode acelerar o tempo de pega da fase CA [3].

As aluminas hidratáveis foram desenvolvidas como alternativa direta ao uso de cimentos refratários, já que o óxido de cálcio oriundo do cimento leva a uma deterioração das propriedades mecânicas dos concretos a altas temperaturas em sistemas contendo sílica.

Tais ligantes hidráulicos podem ser compostos por uma variedade de fases de transição da alumina. São geralmente produzidos pela calcinação rápida da gibsita, resultando principalmente na alumina-rho ( $\rho$ ), que apresenta baixa cristalinidade e alta área superficial. A capacidade ligante deste material deriva da sua fácil re-hidratação quando em contato com a água a temperatura ambiente [4]:

$$
\rho-\mathrm{Al}_{2} \mathrm{O}_{3}+\mathrm{H}_{2} \mathrm{O} \rightarrow \mathrm{Al}_{2} \mathrm{O}_{3} \cdot 3 \mathrm{H}_{2} \mathrm{O}+\mathrm{Al}_{2} \mathrm{O}_{3}(1 \approx 2) \mathrm{H}_{2} \mathrm{O}
$$

Nos primeiros estágios da hidratação uma espessa camada de alumina gel é formada e, com o decorrer da reação, parte deste gel é transformado em boemita $\left(\mathrm{Al}_{2} \mathrm{O}_{3} 1 \approx 2 \mathrm{H}_{2} \mathrm{O}\right)$ e principalmente baierita $\left(\mathrm{Al}_{2} \mathrm{O}_{3} 3 \mathrm{H}_{2} \mathrm{O}\right)$. Os cristais interligados de baierita e o gel conferem resistência mecânica a verde aos refratários por meio do preenchimento de poros e redução dos defeitos superficiais. Tal cristalização também favorece a formação de estruturas na superfície dos agregados, unindo os grãos adjacentes à matriz circundante [5].

A cinética do processo de hidratação pode ser significativamente modificada em função do tipo de ligante hidráulico, da temperatura e de materiais finos (matriz) ou aditivos inorgânicos presentes no concreto. Neste contexto, esse trabalho teve como objetivo avaliar a influência dessas variáveis no processo de hidratação por meio de medidas de temperatura, condutividade elétrica e ensaios reológicos oscilatórios em função do tempo.

\section{MATERIAIS E MÉTODOS}

Os ligantes hidráulicos avaliados foram as aluminas hidratáveis Alphabond 300 e 500 (Almatis-US) e os cimentos de aluminato de cálcio CA14M e CA270 (AlmatisUS), Secar 71 e Secar Plenium (Lafarge AluminatesFrança), cujas características são apresentadas na Tabela I. Os finos utilizados (Tabela II) foram o Esy Pump 1000 e as aluminas calcinadas A17-NE e A-1000SG (Almatis-US). Tais materiais finos normalmente constituem as matrizes de concretos refratários sendo utilizados isoladamente (Esy Pump ou A17-NE) ou combinados A17-NE:A1000 (93:7\%). Como aditivos inorgânicos foram selecionados o carbonato de lítio $\left(\mathrm{Li}_{2} \mathrm{CO}_{3}\right)$ e o carbonato de sódio $\left(\mathrm{Na}_{2} \mathrm{CO}_{3}\right)$ da Labsynth-Brasil.

Para avaliação da influência da temperatura $\left(30\right.$ ou $\left.50^{\circ} \mathrm{C}\right)$ ou de aditivos inorgânicos no processo de hidratação, foram preparadas suspensões aquosas dos diferentes ligantes hidráulicos contendo $16,8 \%$ em volume de sólidos $(37-40 \%$ em peso) na ausência ou na presença de $\mathrm{Li}_{2} \mathrm{CO}_{3}$ e $\mathrm{Na}_{2} \mathrm{CO}_{3}$ $(0,01$ e $0,25 \%$ em peso do ligante para a temperatura de $30{ }^{\circ} \mathrm{C}$ ), respectivamente. Para verificar a influência da matriz foram preparadas suspensões aquosas dos diferentes

Tabela I - Composições químicas e propriedades físicas dos ligantes estudados.

[Table I - Chemical compositions and physical properties of the binders studied.]

\begin{tabular}{ccccccc}
\hline $\begin{array}{c}\text { Fases } \\
(\%)\end{array}$ & $\begin{array}{c}\text { Alphabond } \\
300\end{array}$ & $\begin{array}{c}\text { Alphabond } \\
500\end{array}$ & CA14M & CA270 & $\begin{array}{c}\text { Secar } \\
71\end{array}$ & Secar Plenium \\
\hline $\mathrm{Al}_{2} \mathrm{O}_{3}$ & 88 & 83 & 72 & 73 & 68 & 82 \\
$\mathrm{CaO}$ & 0,1 & 0,6 & 27 & 26 & 31 & 18 \\
$\mathrm{SiO}_{2}$ & 0,3 & 0,3 & 0,3 & 0,3 & 0,8 & 0,3 \\
$\mathrm{Na}_{2} \mathrm{O}$ & 0,6 & 0,3 & 0,3 & 0,3 & 0,5 & 0,7 \\
$25-250^{\circ} \mathrm{C}^{*}$ & 4,5 & 6,5 & - & - & - & - \\
$250-1100^{\circ} \mathrm{C}^{*}$ & 6,5 & 9,2 & - & - & - & - \\
Densidade $\left(\mathrm{g} / \mathrm{cm}^{3}\right)$ & 3,20 & 3,20 & 2,96 & 3,15 & 2,98 & 3,25 \\
$\mathrm{ASE} \mathrm{BET}\left(\mathrm{m}^{2} / \mathrm{g}\right)$ & 194 & 165 & 1,87 & 1,88 & 1,17 & 5,78 \\
$\mathrm{D}_{50}(\mu \mathrm{m})$ & 3,3 & 6,2 & 9,4 & 7,8 & 13 & 10 \\
\hline
\end{tabular}

*Perda de massa no intervalo de temperatura. 
Tabela II - Composições químicas e propriedades físicas dos materiais finos utilizados.

[Table II - Chemical compositions and physical properties of the fines materials tested.]

\begin{tabular}{cccc}
\hline $\begin{array}{c}\text { Fases } \\
(\%)\end{array}$ & $\begin{array}{c}\text { Esy } \\
\text { Pump 1000 }\end{array}$ & A17-NE* & A-1000SG* \\
\hline $\mathrm{Al}_{2} \mathrm{O}_{3}$ & 99,4 & 99,8 & 99,8 \\
$\mathrm{CaO}$ & - & 0,02 & 0,02 \\
$\mathrm{SiO}$ & 0,10 & 0,03 & 0,03 \\
$\mathrm{Na}_{2} \mathrm{O}$ & 0,30 & 0,10 & 0,07 \\
Densidade $\left(\mathrm{g} / \mathrm{cm}^{3}\right)$ & 3,99 & 3,94 & 3,95 \\
$\mathrm{ASE} \mathrm{BET}\left(\mathrm{m}^{2} / \mathrm{g}\right)$ & 3,3 & 2,9 & 8,4 \\
$\mathrm{D}_{50}(\mu \mathrm{m})$ & 2,6 & 4,0 & 0,57 \\
\hline
\end{tabular}

*A matriz A17-NE:A-1000SG (93:7) apresenta ASE $=3,28 \mathrm{~m}^{2} / \mathrm{g} e$ teor de $\mathrm{Na}_{2} \mathrm{O}$ de 0,098\%.

ligantes hidráulicos contendo $50 \%$ em peso dos materiais finos, resultando numa concentração de sólidos de $26 \%$ em volume.

Imediatamente após a mistura com auxílio de um agitador magnético, as suspensões foram submetidas a medidas de temperatura e a ensaios reológicos oscilatórios.

As medidas de temperatura foram monitoradas em função do tempo, com o auxílio de um termopar conectado a um sistema automático de aquisição de dados (ESA 9800, Matec Applied Sciences - UK). Esse sensor foi inserido nas suspensões de ligante sendo as medidas imediatamente iniciadas. O sistema para o controle de temperatura foi especialmente desenvolvido para permitir medidas nas temperaturas desejadas $\left(30\right.$ ou $\left.50{ }^{\circ} \mathrm{C}\right)$.

Os ensaios reológicos oscilatórios em função do tempo foram realizados utilizando-se o equipamento Rheostress 300 da ThermoHaake. As medidas reológicas obtidas nesses ensaios são o módulo de estocagem G', que representa as características elásticas da suspensão, e o módulo de perda G”, que está associado às propriedades viscosas do material. Tais medidas devem ser realizadas na região onde o material apresenta um regime viscoelástico linear, ou seja, para uma combinação de tensão $(\tau)$ e freqüência $(f)$ em que $G$ ' permaneça constante preservando assim a estrutura do material em curtos intervalos de tempo. Dessa forma, testes preliminares com variação de $\tau$ e f foram realizados para cada tipo de ligante a fim de determinar os valores a serem adotados em cada caso. Tais ensaios também foram executados com controle de temperatura em 30 ou $50{ }^{\circ} \mathrm{C}$.

Adicionalmente, medidas de condutividade elétrica em função do tempo foram realizadas para todos os tipos de ligantes, utilizando-se um eletrodo de condutividade (ESA 9800, Matec Applied Sciences - UK). Suspensões aquosas dos ligantes foram preparadas com um teor de sólidos de 8,4\% em volume para a realização das medidas nas temperaturas de 30 ou $50{ }^{\circ} \mathrm{C}$, e também na presença de $0,01 \%$-p $\mathrm{Li}_{2} \mathrm{CO}_{3}$ para a temperatura de $30{ }^{\circ} \mathrm{C}$. Suspensões aquosas das diferentes matrizes foram preparadas com um teor de sólidos de $16,8 \%$ em volume para medidas de condutividade a $50{ }^{\circ} \mathrm{C}$.

\section{RESULTADOS E DISCUSSÃO}

A hidratação do cimento é caracterizada pela soma das hidratações de cada um dos seus componentes. Durante a hidratação das fases mais reativas certa quantidade de calor é liberada (reação exotérmica) fazendo com que a temperatura da pasta de cimento aumente, assegurando assim a reação até mesmo das fases mais inertes [6]. Geralmente, na hidratação de um cimento de aluminato de cálcio se verifica, inicialmente, uma pequena geração de calor. Segue um período dormente após o qual a precipitação de hidratos ocorre e um correspondente aumento na evolução de calor é observado. As reações de re-hidratação das aluminas de transição transformando-se parcialmente em gel e posteriormente em diferentes hidróxidos de alumínio, também são exotérmicas [7]. Dessa forma, por meio da introdução de um termopar na pasta de ligante hidráulico o aumento de temperatura pode ser detectado e usado como medida da reação dos cimentos e aluminas hidratáveis.

As medidas do módulo de estocagem G', permitem verificar a influência do fenômeno de hidratação nas interações coloidais e comportamento de pega inicial [8]. Com a consolidação da suspensão é verificado um aumento de G' e uma dispersão dos valores de G', a medida que o módulo de estocagem se aproxima de um valor constante (aproximadamente $10^{6} \mathrm{~Pa}$ ).
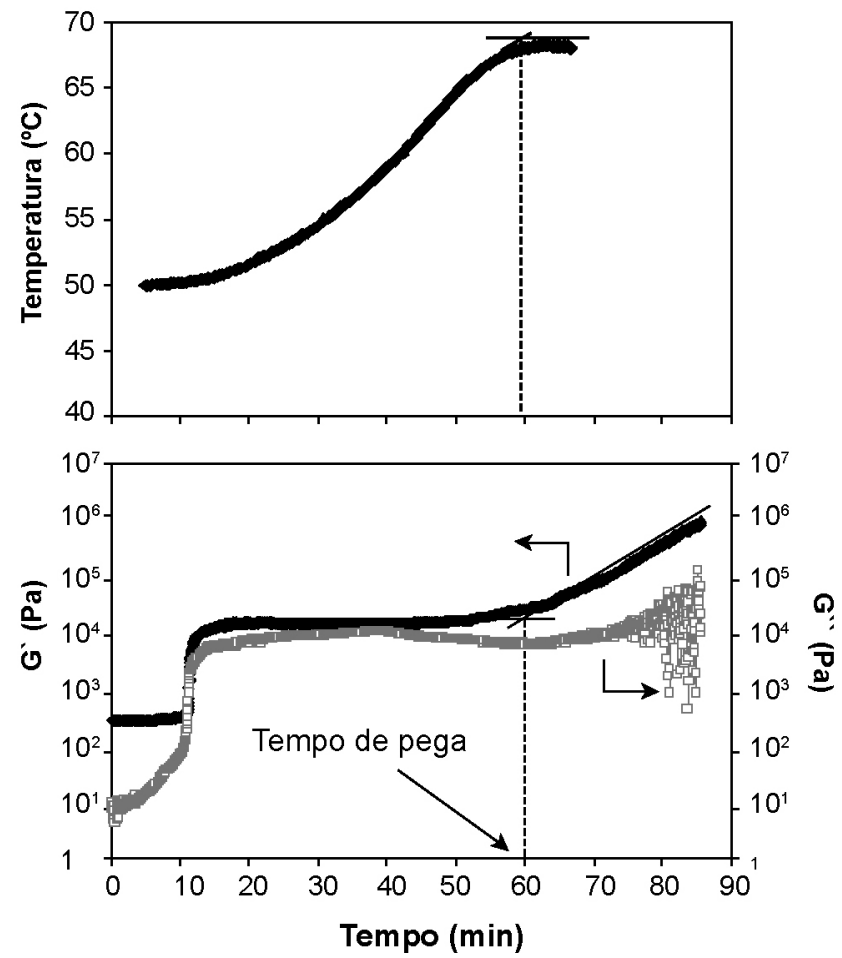

Figura 1: Medidas de temperatura e ensaios reológicos oscilatórios, em função do tempo, para suspensões aquosas de cimento de aluminato de cálcio CA14M, mantendo-se a temperatura do banho térmico em $50{ }^{\circ} \mathrm{C}$.

[Figure 1: Temperature measurements and oscillatory rheological tests as a function of the time for aqueous suspensions of calcium aluminate cement $(\mathrm{CA1} 4 \mathrm{M})$ at $50^{\circ} \mathrm{C}$.] 
Neste trabalho, medidas de temperatura e ensaios reológicos oscilatórios foram realizados para suspensões de todos os tipos de ligantes puros ou quando preparadas na presença de matriz ou aditivos inorgânicos. Um exemplo é apresentado na Fig. 1 para as suspensões do cimento CA14M puro. Nesta figura são indicadas as tangentes das curvas de temperatura e G', cujas intersecções foram coincidentes e tomadas como o tempo de pega ou endurecimento do cimento. Do mesmo modo, foram determinados os tempos de pega utilizados para interpretação de todos os resultados relacionados à influência das variáveis sobre o processo de hidratação dos diferentes ligantes.

\section{Avaliação quanto ao tipo de ligante hidráulico e da temperatura}

A precipitação dos hidratos envolve um processo lento de nucleação sendo que para a formação dos primeiros germes dos cristais dos hidratos é necessária a superação de uma barreira de energia de ativação $\left(\mathrm{E}_{\mathrm{a}}\right)$. A partir da formação dos primeiros germes de hidratos a velocidade do processo passa a não ser mais limitada pela $\mathrm{E}_{\mathrm{a}}$, uma vez que a precipitação passa a ocorrer por meio do crescimento deles. Dessa maneira, íons de $\mathrm{Ca}^{2+} \mathrm{e} \mathrm{Al}(\mathrm{OH})_{4}^{-}$são retirados da solução, proporcionando a retomada da dissolução das partículas de cimento, o que possibilita um contínuo crescimento dos hidratos.

A nucleação é um fenômeno dinâmico que pode ser descrito considerando que os íons $\mathrm{Ca}^{2+}$ e $\mathrm{Al}(\mathrm{OH})_{4}^{-}$e as moléculas de água estão em constante movimento, chocando-se uns contra os outros com uma freqüência que aumenta com a temperatura e com a concentração das espécies. Dessa forma ligações entre essas espécies ocorrem aleatoriamente sendo constantemente formadas e rompidas. No entanto, em um certo instante, um conjunto de espécies pode permanecer unido por um tempo suficiente tal que o aumento de seu tamanho seja favorecido pela ligação de outras espécies. Assim que se alcança um determinado tamanho crítico se verifica a formação de um germe estável. Tal processo, corresponde a idéia da superação da barreira $\mathrm{E}_{\mathrm{a}}$. Dessa maneira, quando um número suficiente de germes estáveis é formado ocorre o seu crescimento, resultando na precipitação dos hidratos de aluminato de cálcio [3].

A temperatura, bem como a concentração dos íons em solução provenientes da dissolução do cimento, além de exercer influência sobre a etapa de nucleação também determina o tipo de hidrato formado. Em baixas temperaturas $\left(5-10{ }^{\circ} \mathrm{C}\right)$, a fase majoritária formada é o hidrato $\mathrm{CAH}_{10}\left(\mathrm{CaO} \cdot \mathrm{Al}_{2} \mathrm{O}_{3} \cdot 10 \mathrm{H}_{2} \mathrm{O}\right)$ que é o menos denso $\left(1,72{\mathrm{~g} . \mathrm{cm}^{-3}}^{-3}\right.$ e o mais solúvel. O hidrato $\mathrm{C}_{2} \mathrm{AH}_{8}(2 \mathrm{CaO}$. $\left.\mathrm{Al}_{2} \mathrm{O}_{3} \cdot 8 \mathrm{H}_{2} \mathrm{O}\right)$ forma-se entre $22-35{ }^{\circ} \mathrm{C}$ e possui valores intermediários de densidade $\left(1,95 \mathrm{~g} . \mathrm{cm}^{-3}\right)$ e de solubilidade. Já o hidrato $\mathrm{C}_{3} \mathrm{AH}_{6}\left(3 \mathrm{CaO} \cdot \mathrm{Al}_{2} \mathrm{O}_{3} \cdot 6 \mathrm{H}_{2} \mathrm{O}\right)$ é o hidrato formado majoritariamente em temperaturas acima de $35{ }^{\circ} \mathrm{C}$, sendo o mais denso $\left(2,52{\mathrm{~g} . \mathrm{cm}^{-3}}^{-3}\right)$ e o menos solúvel dos três $[3,9]$.

Sendo assim, a energia de ativação para a nucleação é maior para o $\mathrm{C}_{3} \mathrm{AH}_{6}$ e menor para o $\mathrm{CAH}_{10}$, isso porque o primeiro forma-se apenas em temperaturas mais altas e em tempos mais longos indicando uma cinética lenta. Já o $\mathrm{CAH}_{10}$ forma-se em temperaturas mais baixas e em tempos mais curtos, indicando uma cinética de formação mais rápida (menor barreira $\mathrm{E}_{\mathrm{a}}$ ).

Com relação a concentração dos íons $\mathrm{Ca}^{2+} \mathrm{e} \mathrm{Al}(\mathrm{OH})_{4}^{-} \mathrm{em}$ solução provenientes da dissolução do cimento, esta depende do teor das diferentes fases que o cimento é constituído $\left(\mathrm{CA}, \mathrm{CA}_{2}, \mathrm{C}_{12} \mathrm{~A}_{7}\right.$ e $\left.\mathrm{Al}_{2} \mathrm{O}_{3}\right)$. Tais fases apresentam diferentes velocidades de hidratação, sendo que a fase $\mathrm{CA}_{2}$ requer um longo tempo para completar sua hidratação. Já a fase $\mathrm{C}_{12} \mathrm{~A}_{7}$, ao contrário, necessita de um tempo curto para se hidratar (dissociação rápida). Dessa forma, quanto maior o teor da fase $\mathrm{C}_{12} \mathrm{~A}_{7}$ presente no cimento, mais rápido ocorrerá a saturação e a precipitação de hidratos. Além disso, a diferença imposta pelo processo de hidratação das diferentes fases anidras está relacionada com a quantidade relativa entre as concentrações de $\mathrm{Ca}^{2+}$ e $\mathrm{Al}(\mathrm{OH})_{4}^{-}$que cada uma delas pode gerar em solução. Como por exemplo, a dissolução da fase $\mathrm{C}_{12} \mathrm{~A}_{7}$ levará a uma solução mais rica em $\mathrm{Ca}^{2+}$.

Consequentemente, a concentração dos íons $\mathrm{Ca}^{2+} \mathrm{e}$ $\mathrm{Al}(\mathrm{OH})_{4}^{-}$em solução permitirá e ditará a fase de hidrato que será formada. A relação entre as concentrações desses íons em solução é convencionalmente representada por C/A que expressa a quantidade de cálcio e de alumínio na forma de $\mathrm{CaO}$ e $\mathrm{Al}_{2} \mathrm{O}_{3}$, respectivamente. Como exemplo, se $\left[\mathrm{Ca}^{2+}\right] /$ $\left[\mathrm{Al}(\mathrm{OH})_{4}^{-}\right]^{2}$ for $0,5, \mathrm{C} / \mathrm{A}$ será igual a 1 .

Sendo assim, o valor de C/A determina qual hidrato será formado em uma determinada temperatura. $\mathrm{O}$ diagrama de solubilidade para o sistema $\mathrm{CaO}-\mathrm{Al}_{2} \mathrm{O}_{3}-\mathrm{H}_{2} \mathrm{O}$, obtido a $25^{\circ} \mathrm{C}$ [10] e ilustrado na Fig. 2, mostra que para valores de C/A próximos a 1, a formação do hidrato $\mathrm{CAH}_{10}$ é favorecida. Com o aumento de $\mathrm{C} / \mathrm{A}$, ou seja, para maiores $\left[\mathrm{Ca}^{2+}\right.$, passase a favorecer a formação de $\mathrm{C}_{2} \mathrm{AH}_{8}$. Já a diminuição de $\mathrm{C} / \mathrm{A}$ (maiores teores de $\mathrm{Al}(\mathrm{OH})_{4}^{-}$) favorece a formação de $\mathrm{AH}_{3}$.

Uma vez que os tipos de hidratos formados apresentam diferentes graus de solubilidade, a formação do tipo menos solúvel implicará numa alta velocidade de precipitação reduzindo o tempo de pega do cimento. Os hidratos formados segundo a Fig. 2, apresentam a seguinte ordem de solubilidade $\mathrm{C}_{2} \mathrm{AH}_{8}<\mathrm{CAH}_{10}<\mathrm{AH}_{3}$ Um raciocínio semelhante pode ser aplicado para se entender o atraso no tempo de pega com a diminuição de C/A para valores menores que 1. Nesse caso o hidrato favorecido é o $\mathrm{AH}_{3}$, que inicialmente tem uma solubilidade maior que $\mathrm{CAH}_{10}$ e por esse motivo requer um maior período de indução (tempo de espera para que se atinja condições para a nucleação).

O tempo de pega avaliado para diferentes tipos de ligantes, em função da temperatura a qual a suspensão foi exposta, são apresentados na Fig. 3. Observa-se que o tempo de pega é significativamente influenciado pela temperatura, sendo este mais longo para a temperatura de $30^{\circ} \mathrm{C}$. Entre os diferentes tipos de ligantes, o Alphabond 300 apresentou o menor tempo de pega em ambas as temperaturas, uma vez que as aluminas hidratáveis apresentam um processo de hidratação bem mais simples e, portanto, mais rápido já a temperatura ambiente. Além disso, a alta área superficial desse ligante facilita a reação com a água. Entretanto o Alphabond 500, apesar de 
também ser uma alumina hidratável com alta área superficial, apresentou o maior tempo de hidratação, mesmo a $50{ }^{\circ} \mathrm{C}$. Tem sido verificado que os aditivos dispersantes comumente utilizados nas formulações dos concretos, apresentam um efeito retardador do processo de hidratação de cimentos, entre outros motivos, pelo fato de serem adsorvidos na superfície das partículas barrando a sua dissociação [11]. O fato do Alphabond 500 , segundo informações do fabricante, apresentar em sua composição um dispersante, este deve estar dificultando sua rehidratação, o que poderia explicar a demora para a verificação da sua pega quando comparado ao Alphabond 300.

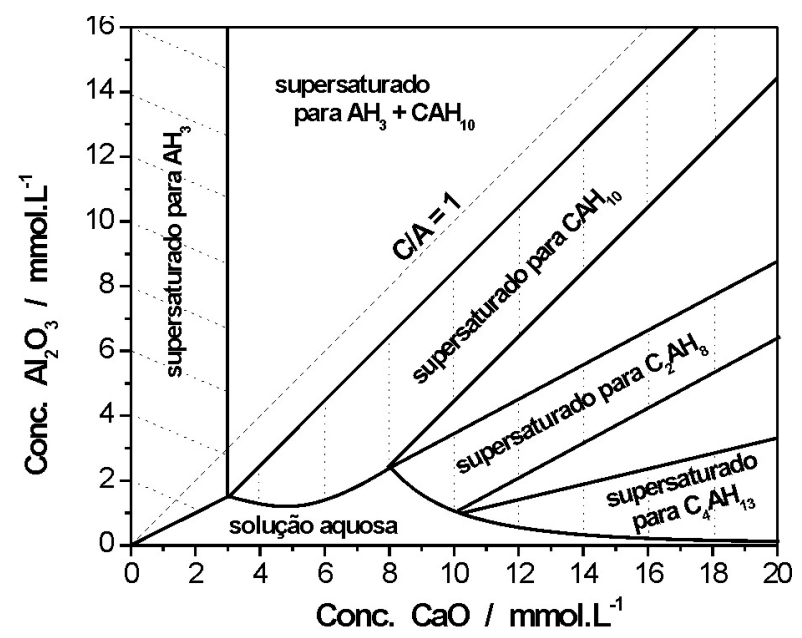

Figura 2: Diagrama de solubilidade para o sistema ternário CaO$\mathrm{Al}_{2} \mathrm{O}_{3}-\mathrm{H}_{2} \mathrm{O}$, obtido a $25^{\circ} \mathrm{C}[10]$.

[Figure 2: Solubility diagram for the ternary system $\mathrm{CaO}-\mathrm{Al}_{2} \mathrm{O}_{3}-$ $\mathrm{H}_{2} \mathrm{O}$ at $25^{\circ} \mathrm{C}$.]

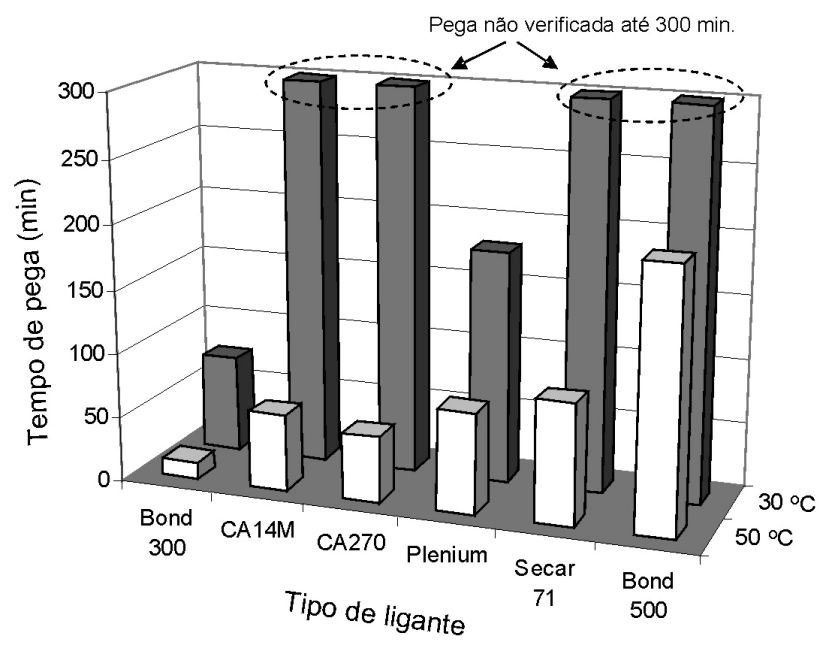

Figura 3: Tempos de pega avaliados por meio de medidas de temperatura e ensaios reológicos oscilatórios, em função do tempo, para suspensões aquosas dos diferentes tipos de ligante mantendose a temperatura do banho térmico em 30 ou $50^{\circ} \mathrm{C}$.

[Figure 3: Setting time evaluated through temperaturemeasurements and oscillatory rheological tests as a function of time for aqueous suspensions of the different binder sources, at 30 or $50^{\circ} \mathrm{C}$.]
Por outro lado, entre os cimentos de aluminato de cálcio, o Secar Plenium apresentou o menor tempo de pega para a temperatura de $30{ }^{\circ} \mathrm{C}$. De acordo com sua composição química (Tabela I) este tipo de cimento apresenta um alto teor de $\mathrm{Al}_{2} \mathrm{O}_{3}$, decorrente provavelmente à adição de alumina de alta área superficial à fase de aluminato de cálcio. Tais partículas de alumina constituem germes para a nucleação de hidratos favorecendo a etapa de precipitação e portanto acelerando a sua pega. Neste caso, o hidrato majoritariamente formado deve ser $\mathrm{O}_{3} \mathrm{H}_{3}$ devido ao menor teor de $\mathrm{CaO}$ deste cimento, resultando no decréscimo de $\mathrm{C} / \mathrm{A}$ (maiores teores de $\left.\mathrm{Al}(\mathrm{OH})_{4}^{-}\right)^{-}$.

Adicionalmente, foram realizadas curvas de condutividade para todos os tipos de ligantes nas temperaturas de 30 ou $50{ }^{\circ} \mathrm{C}$ (Fig. 4). A dissolução dos íons $\mathrm{Ca}^{2+} \mathrm{e} \mathrm{Al}(\mathrm{OH})_{4}^{-}$das partículas de cimento em água causa um aumento da condutividade elétrica das suspensões aquosas de cimento no estágio inicial do processo de hidratação. Posteriormente verifica-se uma queda de condutividade relacionada a precipitação de hidratos [12]. Com relação as aluminas hidratáveis, não se verifica o mesmo comportamento uma vez que seu processo de hidratação não envolve a dissolução de íons e precipitação de fases hidratadas. Cabe ressaltar que os tempos de pega observados para os diferentes tipos de ligantes são distintos dos apresentados na Fig. 3, uma vez que os testes foram realizados sob agitação ao contrário dos ensaios de temperatura e oscilatórios. A agitação deve contribuir para a nucleação, ou seja, para o aumento das colisões entre os íons $\mathrm{Ca}^{2+}$ e $\mathrm{Al}(\mathrm{OH})_{4}^{-}$e as moléculas de água, verificando-se redução nos tempos de pega mesmo a $30^{\circ} \mathrm{C}$.

As curvas de condutividade (Fig. 4) mostram que para o caso do Secar Plenium o aumento de condutividade (dissolução) é mais lento comparado aos outros tipos de cimento. Isso pode ser justificado pelo seu menor teor de $\mathrm{CaO}$ indicando que este tipo de cimento é constituído por fases menos reativas $\left(\mathrm{CA}_{2}\right.$ e $\left.\mathrm{CA}\right)$. Esperava-se também que a dissolução prosseguisse até que um valor constante de condutividade fosse alcançado permanecendo neste patamar (processo de nucleação e crescimento) por um período de tempo maior que o observado para os demais cimentos devido à formação majoritária do hidrato mais solúvel $\left(\mathrm{AH}_{3}\right)$. Entretanto, observa-se que tão logo a condutividade atinge um valor máximo é seguida de uma queda (precipitação). Isso confirma a idéia que $\mathrm{o} \mathrm{Al}_{2} \mathrm{O}_{3}$ deve estar atuando como germe para a nucleação do $\mathrm{AH}_{3}$ acelerando a sua precipitação.

Já com o aumento da temperatura para $50{ }^{\circ} \mathrm{C}$, observa-se decréscimo do tempo de pega para todos os tipos de cimentos, tanto por meio dos ensaios de temperatura e oscilatórios (Fig. 3), como através das medidas de condutividade (Fig. 4). Isso ocorre porque a formação do hidrato menos solúvel $\mathrm{C}_{3} \mathrm{AH}_{6}$ é favorecida em temperaturas acima de $35^{\circ} \mathrm{C}$, facilitando a sua precipitação. Entretanto, o Secar Plenium que a $30{ }^{\circ} \mathrm{C}$ apresentou um tempo de pega significativamente menor que os demais cimentos, não apresentou o mesmo comportamento a $50^{\circ} \mathrm{C}$. Isso pode ser explicado pelo seu menor teor de $\mathrm{CaO}$, fazendo com que a relação entre os íons responsáveis pela produção dos hidratos resulte numa menor probabilidade de formação da espécie menos solúvel a $50{ }^{\circ} \mathrm{C}$. Este resultado 
evidencia que não basta o aumento da temperatura propiciar a formação do hidrato menos solúvel, deve-se também haver uma concentração de íons em solução que o favoreça.

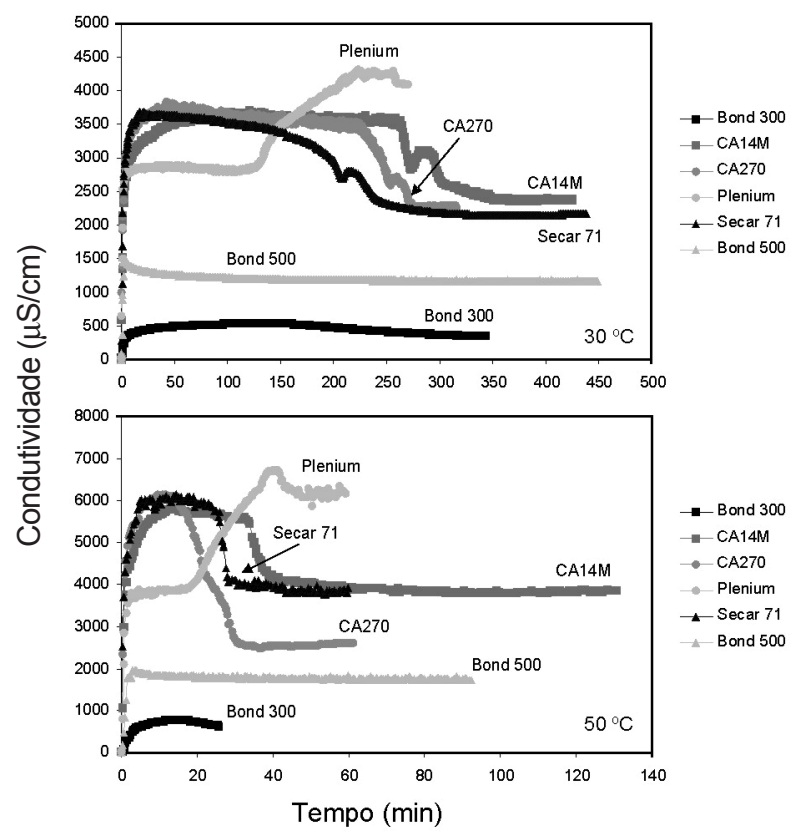

Figura 4: Curvas de condutividade elétrica em função do tempo obtidas para suspensões aquosas dos diferentes tipos de ligantes puros para as temperaturas de 30 e $50{ }^{\circ} \mathrm{C}$.

[Figure 4: Electrical conductivity curves as a function of time obtained for aqueous suspensions of the different binder sources, at 30 or $50^{\circ} \mathrm{C}$.]

Avaliação quanto ao tipo de ligante hidráulico e aditivo inorgânico

Os tempos de precipitação de hidratos também foram avaliados para diferentes tipos de ligantes hidráulicos na presença de aditivos inorgânicos para a temperatura de $30^{\circ} \mathrm{C}$. Os resultados obtidos por meio de medidas de temperatura ou ensaios reológicos para $\mathrm{Li}_{2} \mathrm{CO}_{3}$ e $\mathrm{Na}_{2} \mathrm{CO}_{3}$ são apresentados nas Figs. 5 e 6, respectivamente.

$\mathrm{Na}$ presença de carbonato de lítio observa-se uma considerável redução no tempo de pega para os cimentos CA14M, CA270 e Secar 71, sendo o seu efeito intensificado com o aumento do teor, como exemplificado para o CA14M. Por outro lado, na presença de carbonato de sódio pouco efeito foi observado até quando o teor foi aumentado de 0,01 para $0,25 \%$. Rodger e Doublé [13] mostraram a influência de sais de cloreto com diferentes cátions e verificaram que nenhum dos cátions testados teve um efeito acelerador comparável ao do lítio. De fato, os compostos químicos mais utilizados como aditivos aceleradores do tempo de pega são os sais de lítio. O interesse nesse composto como aditivo acelerador deve-se a sua grande eficiência, já que com teores da ordem de $0,01 \%$ obtém-se grande efeito no processo de endurecimento do cimento $[14,15]$. De uma maneira geral, aditivos aceleradores agem alterando o processo de precipitação dos hidratos de forma a diminuir o tempo necessário a nucleação, que ocorrendo mais facilmente possibilita que a precipitação aconteça assim que o estado de saturação da solução seja atingido. A presença de $\mathrm{Li}^{+}$ em solução pode proporcionar a formação de compostos insolúveis do tipo $\mathrm{LiAl}(\mathrm{OH})_{4}$, retirando íons $\mathrm{Al}(\mathrm{OH})_{4}^{-}$da solução $[14,15]$. Dessa forma, os sais de lítio atuam na reação de hidratação do cimento aumentando a relação C/A, favorecendo a formação de hidratos menos solúveis que irão precipitar mais facilmente.

Adicionalmente, a precipitação desses compostos insolúveis de lítio é mais rápida do que a precipitação dos hidratos de aluminato de cálcio e apresentam estrutura cristalina necessária para servir de germes para a nucleação do hidrato $\mathrm{C}_{2} \mathrm{AH}_{8}$, independente da temperatura. Isso faz com que a barreira de energia necessária à nucleação desse hidrato passe a não existir mais. Tais fenômenos podem explicar a considerável redução no tempo de pega observada para os cimentos CA14M, CA270 e Secar 71. Entretanto, o efeito acelerador do carbonato de lítio foi menos pronunciado para o Secar Plenium. O fato deste tipo de cimento apresentar um menor teor de $\mathrm{CaO}$ (Tabela I) faz com que se tenha menos íons $\mathrm{Ca}^{2+}$ provenientes da dissolução da fase anidra resultando em uma menor tendência a formação do hidrato $\mathrm{C}_{2} \mathrm{AH}_{8}$, que é aquele favorecido quando na presença de $\mathrm{Li}_{2} \mathrm{CO}_{3}$. Dessa forma, assim como discutido para o aumento da temperatura, não basta existir germes que facilitem a precipitação de um determinado hidrato, mas deve também haver uma concentração de íons em solução suficientes para a sua formação.

O significativo efeito acelerador do tempo de pega dos cimentos observado para o carbonato de lítio, com exceção do Secar Plenium, também foi verificado por medidas de condutividade (Fig. 7).

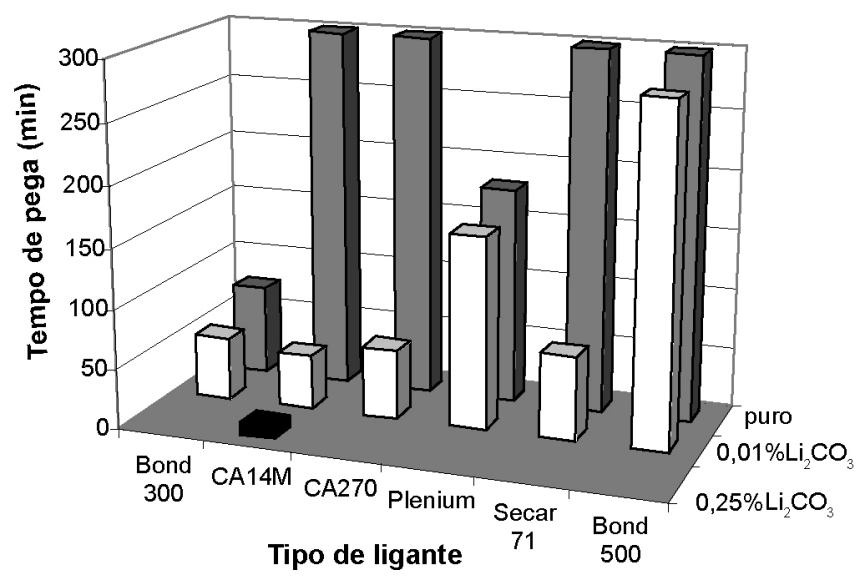

Figura 5: Tempos de pega avaliados por meio de medidas de temperatura e ensaios reológicos oscilatórios, em função do tempo, para suspensões aquosas dos diferentes tipos de ligante na presença de 0,01 ou $0,25 \%$-p de $\mathrm{Li}_{2} \mathrm{CO}_{3}$ para a temperatura de $30^{\circ} \mathrm{C}$.

[Figure 5: Setting time evaluated through temperature measurements and oscillatory rheological tests as a function of time for aqueous suspensions of the different binder sources in the presence of $\mathrm{Li}_{2} \mathrm{CO}_{3}$ (0.01 or $0.25 w t \%)$, at $30^{\circ} \mathrm{C}$.] 


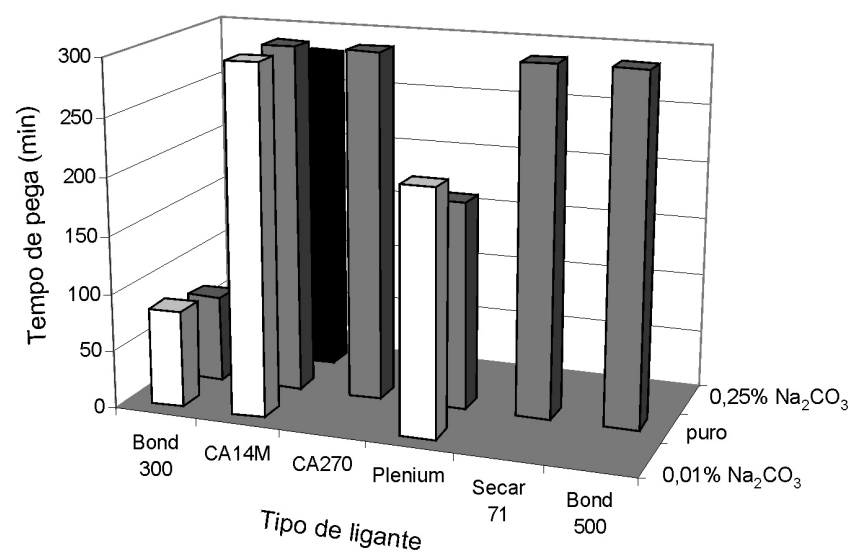

Figura 6: Tempos de pega avaliados por meio de medidas de temperatura e ensaios reológicos oscilatórios, em função do tempo, para suspensões aquosas dos diferentes tipos de ligante na presença de 0,01 ou $0,25 \%$-p de $\mathrm{Na}_{2} \mathrm{CO}_{3}$ para a temperatura de $30^{\circ} \mathrm{C}$.

[Figure 6: Setting time evaluated through temperature measurements and oscillatory rheological tests as a function of time for aqueous suspensions of the different binder sources in the presence of $\mathrm{Na}_{2} \mathrm{CO}_{3}(0.01$ or $0.25 w t \%)$, at $30^{\circ} \mathrm{C}$.]

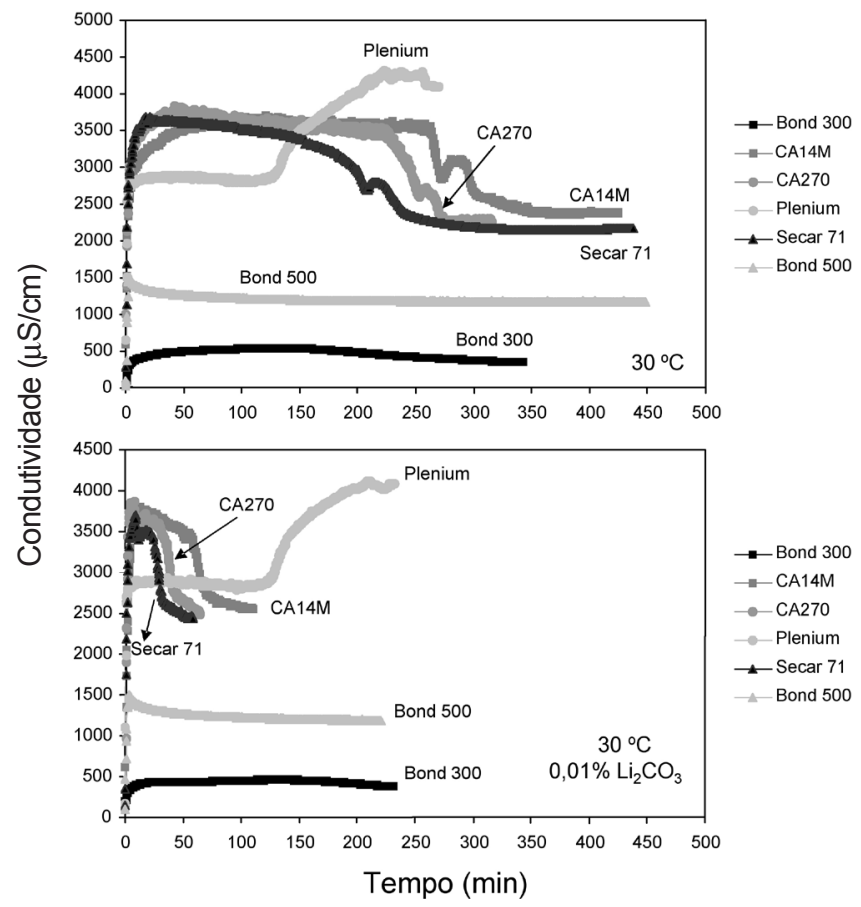

Figura 7: Curvas de condutividade elétrica em função do tempo obtidas para suspensões aquosas dos diferentes tipos de ligantes puros ou na presença de 0,01\%-p de $\mathrm{Li}_{2} \mathrm{CO}_{3}$ para a temperatura de $30^{\circ} \mathrm{C}$.

[Figure 7: Electrical conductivity curves as a function of time obtained for aqueous suspensions of the different binder sources with or no $\mathrm{Li}_{2} \mathrm{CO}_{3}(0.01 \mathrm{wt} \%)$, at $30^{\circ} \mathrm{C}$. ]

Avaliação quanto à influência da matriz no processo de hidratação

A hidratação do cimento pode ser significativamente modificada na presença de alumina [16]. O período de indução (tempo de espera para que se atinja condições para a nucleação) é encurtado pela adição de partículas de alumina à suspensão de cimento uma vez que essas constituem sítios de nucleação que eliminam a barreira de energia necessária para nucleação do hidrato. Este encurtamento do período de indução e conseqüente decréscimo do tempo de pega, são observados para a mistura de todos os tipos de cimento com as diferentes matrizes, como apresentado na Fig. 8. Esses resultados reforçam a idéia de que a presença de alumina no Secar Plenium seja responsável pela aceleração da sua pega a $30^{\circ} \mathrm{C}$.

Entretanto, esta redução no tempo depende da área superficial específica (ASE) da alumina presente na pasta de cimento. Quanto maior a ASE da alumina mais pronunciado é o encurtamento do período de indução durante o processo de hidratação [17]. Quando comparado a ASE das diferentes matrizes avaliadas (Tabela II) observase valores bastante próximos. Isso indica que alguma outra diferença característica das diferentes matrizes deve ser predominante.

De fato, trabalhos têm mostrado a adicional influência do teor de $\mathrm{Na}_{2} \mathrm{O}$ das aluminas no tempo de indução. Se o teor de $\mathrm{Na}_{2} \mathrm{O}$ aumenta, a precipitação de hidratos é acelerada [16, 17]. Considerando aluminas com ASE similares, aquelas com maior teor de $\mathrm{Na}_{2} \mathrm{O}$ resultam nos mais curtos períodos de indução durante a hidratação da sua mistura com cimento.

A Tabela II também apresenta os valores do teor de $\mathrm{Na}_{2} \mathrm{O}$ para as diferentes matrizes. A alumina Esy Pump possui o maior teor deste óxido, resultando nos menores valores de tempo de pega. Isso pode ser entendido visto que o sódio liberado em solução pode atuar diminuindo a quantidade de íns $\mathrm{Al}(\mathrm{OH})_{4}^{-}$por meio da formação de $\mathrm{NaAl}(\mathrm{OH})_{4}$, causando assim um aumento da relação $\mathrm{C} / \mathrm{A}$ o que favorece a precipitação

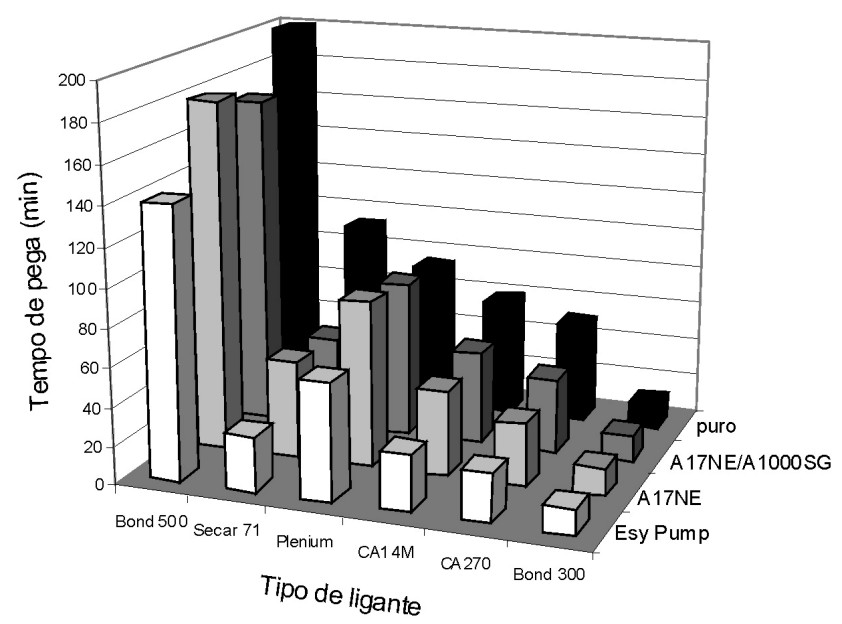

Figura 8: Tempos de pega avaliados por meio de medidas de temperatura em função do tempo, para suspensões aquosas dos diferentes tipos de ligantes puros ou na presença de diferentes finos (50\%-p do ligante), para a temperatura de ensaio de $50{ }^{\circ} \mathrm{C}$.

[Figure 8: Setting time evaluated through temperature measurements as a function of time for aqueous suspensions of the different binder sources containing or not fines (50wt.\% of binder) at $50^{\circ} \mathrm{C}$.] 


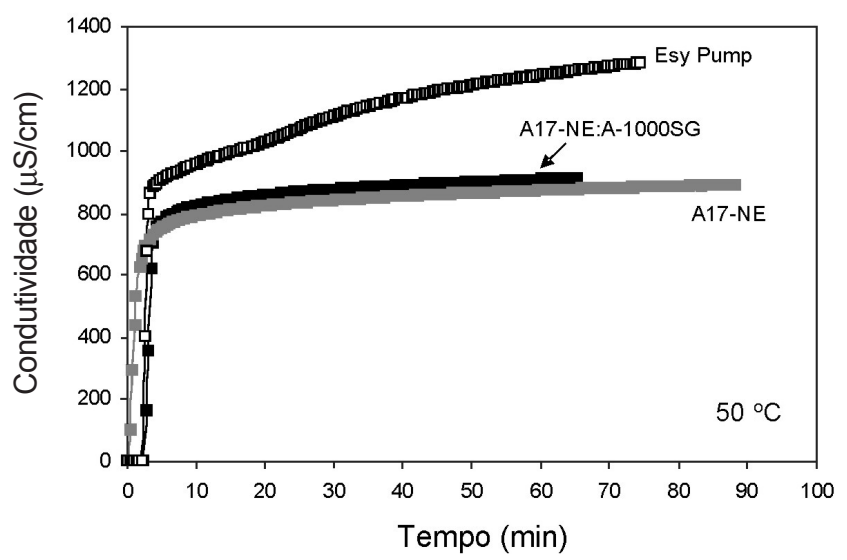

Figura 9: Curvas de condutividade elétrica em função do tempo obtidas para suspensões aquosas das diferentes matrizes para a temperatura de $50{ }^{\circ} \mathrm{C}$.

[Figure 9: Electrical conductivity curves as a function of time obtained for aqueous suspensions of the different fines, at $50^{\circ} \mathrm{C}$. I

do hidrato menos solúvel. A presença de um maior teor de $\mathrm{Na}^{+}$ em solução, quando utilizado a Esy Pump, foi confirmada pelo significativo aumento de condutividade quanto comparado as demais matrizes, como pode ser visto na Fig. 9.

Para o caso do Secar Plenium somente foi verificado decréscimo do tempo de pega para o sistema mais rico em sódio, ou seja, na presença de Esy Pump. Isso porque o decréscimo pronunciado na quantidade íons $\mathrm{Al}(\mathrm{OH})_{4}{ }_{4}^{-}$deve ter resultado em uma quantidade relativa entre as concentrações de $\mathrm{Ca}^{2+}$ e $\mathrm{Al}(\mathrm{OH})_{4}{ }^{-}$tal que favoreceu a formação do hidrato menos solúvel $\mathrm{C}_{3} \mathrm{AH}_{6}$, uma vez que a temperatura de ensaio foi de $50{ }^{\circ} \mathrm{C}$.

Uma exceção foi verificada para o caso do Alphabond 500. Observa-se na Fig. 8 um decréscimo do tempo de pega quando este tipo de alumina de transição é hidratado na presença de matriz. Como discutido anteriormente, a presença de um dispersante na composição do Alphabond 500 dificulta sua re-hidratação. A possível formação de um composto solúvel entre o dispersante e o sódio poderia explicar a liberação da superfície das partículas para a reação com a água.

\section{CONCLUSÃO}

A temperatura, bem como, a concentração dos íons em solução provenientes da dissolução do cimento, além de exercerem influência sobre a etapa de nucleação também determina o tipo de hidrato formado. O tempo de pega é significativamente reduzido com o aumento da temperatura de 30 para $50{ }^{\circ} \mathrm{C}$, uma vez que a fase majoritária formada é o hidrato menos solúvel $\mathrm{C}_{3} \mathrm{AH}_{6}$, facilitando a sua precipitação. Entretanto, os resultados apresentados neste trabalho mostraram que não basta o aumento da temperatura propiciar a formação do hidrato menos solúvel, deve-se também haver uma concentração de íons $\mathrm{Ca}^{2+}$ e $\mathrm{Al}(\mathrm{OH})_{4}^{-}$em solução que o favoreça. A precipitação de um determinado hidrato depende do teor e do tipo de fase que o cimento é constituído uma vez que tais fases apresentam diferentes velocidades de hidratação e determinam a quantidade relativa entre as concentrações de $\mathrm{Ca}^{2+}$ e $\mathrm{Al}(\mathrm{OH})_{4}^{-}$.

Quanto a influência do aditivo inorgânico, a presença de $\mathrm{Li}^{+}$em solução pode proporcionar a formação de compostos insolúveis do tipo $\mathrm{LiAl}(\mathrm{OH})_{4}$, retirando íons $\mathrm{Al}(\mathrm{OH})_{4}^{-}$da solução. A precipitação desses compostos é mais rápida do que a precipitação dos hidratos de aluminato de cálcio e apresentam estrutura cristalina necessária para servir de germes para a nucleação do hidrato $\mathrm{C}_{2} \mathrm{AH}_{8}$, acelerando a pega dos cimentos. Entretanto, o efeito acelerador do carbonato de lítio foi menos pronunciado para o Secar Plenium, uma vez que seu menor teor de $\mathrm{CaO}$ resulta em uma menor tendência da formação do hidrato $\mathrm{C}_{2} \mathrm{AH}_{8}$. Dessa forma, não basta existir germes que facilitem a precipitação de um determinado hidrato, mas deve também haver uma quantidade relativa de íons que permita a sua formação.

Já quanto à influência da matriz, as partículas adicionadas na suspensão de cimento constituem sítios de nucleação fazendo com que a barreira de energia necessária à nucleação do hidrato passe a não existir mais, encurtando o período de indução. Neste trabalho, a redução do tempo de pega foi principalmente atribuída ao teor de $\mathrm{Na}_{2} \mathrm{O}$ das diferentes matrizes. O aumento do sódio liberado em solução favorece a precipitação do hidrato menos solúvel uma vez que diminui a quantidade de íons $\mathrm{Al}(\mathrm{OH})_{4}^{-}$por meio da formação de $\mathrm{NaAl}(\mathrm{OH})_{4}$.

\section{AGRADECIMENTOS}

A Almatis e a Lafarge Aluminates pelo fornecimento das matérias-primas e à FAPESP pelo suporte financeiro.

\section{REFERÊNCIAS}

[1] T. A. Bier, N. E. Bunt, C. Parr, "Calcium aluminate bonded castables: their advantages and applications", Proc. Latin-American Assoc. Refractory Manufacturers Meeting, Buenos Aires, ALAFAR (1996) 75-84.

[2] K. M. Parker, J. H. Sharp, "Refractory calcium aluminate cements", Trans. J. Brit. Ceram. Soc. 81 (1982) 35-42.

[3] C. M. George, "Aspects of calcium aluminate cement hydration", Refractories Symp., The Am. Ceram. Soc., St. Louis, USA (1994) 1-21.

[4] W. Ma, P. W. Brown, "Mechanisms of reaction of hydratable aluminas", J. Am. Ceram. Soc. 82, 2 (1999) 453-456.

[5] Y. Hongo, " $\rho$-Alumina bonded castable refractories", Taikabutsu Overseas 9, 1 (1988) 35-38.

[6] C. Alt, L. Wong, C. Parr, "Measuring castable rheology by exothermic profile", Refractories Applic. News 8, 2 (2003) 15-18. [7] W. Mista, J. Wrzyszcz, "Rehydration of transition aluminas obtained by flash calcination of gibbsite", Thermochim. Acta 331 (1999) 67-72.

[8] G. H. Kirby, J. A. Lewis, “Comb polymer architecture effects on the rheological property evolution of concentrated cement suspensions", J. Am. Ceram. Soc. 87, 9 (2004) 1643-1652. 
[9] C. D. Parr, C. Alt, C. Wohrmeyer, E. Nonnet, "Calcium aluminate cement hydration and its influence on the placing and high temperature properties of reduced cement castable systems", Proc. $39^{\text {th }}$ Annual Symp. Refractories, St. Louis, USA (2003).

[10] D. Sorrentino, F. Sorrentino, M. George, "Mechanisms of hydration of calcium aluminate cements", Mater. Sci. Concrete IV, Ed. J. Skalny, S. Mindess, The Am. Ceram. Soc., Westerville, OH, USA.

[11] C. Jolicoeur, M. A. Si, "Chemical admixture-cement interactions: phenomenology and physico-chemical concepts", Cement Concrete Composites 20 (1998) 87-101. [12] T. A. Bier, A. Mathieu, B. Espinosa, J. P. Bayoux, "The use of conductimetry to characterize the reactivity of calcium aluminate cements", Proc. UNITECR 93 (Unified International Technical Conference on Refractories), S. Paulo, SP (1993) 705-716.

[13] S. A. Rodger, D. D. Double, "The chemistry of hydration of high alumina cement in the presence of accelerating and retarding admixtures", Cement and Concrete Research 14
(1984) 73-82.

[14] B. R. Currell, R. Grzeskowlak, H. G. Midgley, J. R. Parsonage, "The acceleration and retardation of set high alumina cement by additives", Cement and Concrete Research 7 (1987) 420-432.

[15] N. Bunt, C. Revais, M. Vialle, "Additives in calcium aluminate cement containing castables", Proc. UNITECR 97 (Unified International Technical Conference on Refractories), New Orleans, USA (1997) 1347-1354.

[16]C.Parr, C. Wohrmeyer, B. Valdelievre, A. Namba, "Effect of formulation parameters upon the strength development of calcium aluminate cement containing castables", J. Techn. Assoc. Refractories, Japan 23, 4 (2003) 231-238.

[17] W. Gebner, S. Mohmel, A. Schmalstieg, A. Rettel, J. P. Bayoux, A. Capmas, A. Mathieu, "On the influence of the specific surface area and $\mathrm{Na}_{2} \mathrm{O}$ content of aluminas on the hydration processes in $\mathrm{CaO} \cdot \mathrm{Al}_{2} \mathrm{O}_{3} / \mathrm{Al}_{2} \mathrm{O}_{3}$ Mixes", Proc. UNITECR 95 (Unified International Conference on Refractories), Kyoto, Japão (1995) 313-320.

(Rec. 12/08/2005, Ac. 28/11/2005) 